\title{
Dark Matter without New Matter Is Compliant with General Relativity
}

\author{
Stéphane Le Corre \\ Ecole Polytechnique Fédérale de Lausanne, Lausanne, Switzerland \\ Email: stephane.lecorre@epfl.ch
}

How to cite this paper: Le Corre, S. (2017) Dark Matter without New Matter Is Compliant with General Relativity. Open Access Library Journal, 4: e3877. https://doi.org/10.4236/oalib.1103877

Received: August 7, 2017

Accepted: September 8, 2017

Published: September 11, 2017

Copyright (๑) 2017 by author and Open Access Library Inc.

This work is licensed under the Creative Commons Attribution International License (CC BY 4.0).

http://creativecommons.org/licenses/by/4.0/

\section{(c) (i) Open Access}

\begin{abstract}
A recent publication revealed unexpected observations about dark matter. In particular, the observed baryonic mass should probably be sufficient to explain the observed rotation curves (i.e. without dark matter) and their observations gave an empirical relation for weak accelerations. This present work demonstrated that the equations of general relativity allow explaining the term of dark matter (without new matter) in agreement with the results of this publication and allow retrieving this empirical relation (observed values and characteristics of this correlation's curve). These observations constrain drastically the possible gravitational potential in the frame of general relativity to explain the term of dark matter. This theoretical solution has already been studied with several unexpected predictions that have recently been observed. For example, an article revealed that early galaxies (ten billion years ago) didn't have dark matter and a more recent paper showed unlikely alignments of galaxies. To finish the main prediction of this solution, it is recalled: the term of dark matter should be a Lense-Thirring effect, around the earth, of around 0.3 and 0.6 milliarcsecond/year.
\end{abstract}

\section{Subject Areas}

Modern Physics, Theoretical Physics

\section{Keywords}

Dark Matter, Galaxies: Formation, Galaxies: Evolution, Gravitation

\section{Introduction}

One of the most important mysteries of astrophysics is the problem of dark matter. This latter component represents at least five times the quantity of the ordinary mass. And until now, this term cannot be explained. We therefore find 
that our theories operate in a highly coherent and precise manner both on our scale and at large astrophysical scales. But as far as large scales are concerned, this coherence and precision is only possible on the condition of making the hypothesis of the existence of this new term of dark matter. A way to solve this problem is to propose new theories (MOND theories for example), but we can note that the term of dark matter, even if it is an ad hoc term, doesn't generate any contradiction inside our current theories. In fact, we could even pretend that this term demonstrates the extraordinary consistency of our current theories because of the multiplicity of the ways to deduce the quantity of this term leading to its more and more coherent and precise measure. Another way is to propose that this term represents a new exotic matter. This explanation is the more shared and certainly the more studied. But until now, no new matter has been directly detected, despite more and more experiences. A third way is to propose an explanation in the frame of current theories. That's the purpose of the present work. The term of dark matter will be explained by a physical phenomenon of general relativity that is generally neglected.

Starting with the publication [1] giving new observations, we will show that combined with general relativity these observations would allow solving the mystery of dark matter. First, we demonstrate that the relativity general in its linearized form (Section 2) allows idealizing the rotational speed at the end of the galaxies, where the gravitational interaction of ordinary matter is very weak (Section 3). Using the results of the publication, we will deduce an important constraint on the characteristic of the possible solution (Section 4). This constraint allows interpreting the term of dark matter (Section 5) as the second component of the general relativity (in its linearized form) which is at the origin of the Lense-Thirring effect. We will recall that this solution has ever been studied and will demonstrate that the expected theoretical values are in great agreement with the observation of [1] (Section 5). As we will see, this term is very weak, explaining why it is not yet measured. But in the next years, experiments could be sufficiently accurate to test this explanation. We will finish with several unexpected predictions that had been proposed in the frame of this solution and that has recently been observed (Section 6).

But before getting to the heart of our explanation, let's recall the results of the following publication [1] that will be one of the keystones of our article:

- statistically the gravitational potential, $g_{o b s}$, deduced from the observed rotation curves of the galaxies and the gravitational potential, $g_{b a r}$, deduced from the observed distribution of the baryonic mass is strongly correlated;

- this potential $g_{b a r}$ is a solution of the Poisson equation;

- the value of the gravitational potential is around $g_{b a r} \sim 10^{-10.5}$;

- the relation of the potential in the weak accelerations is $g_{\text {obs }} \propto \sqrt{g_{\text {bar }}}$;

- the correlation's curve deviates from the line of unity for values smaller than around $g_{b a r} \sim 10^{-10}$.

As mentioned in [1], this result implies that it is strongly likely that the ob- 
served baryonic mass must be sufficient to explain the observed rotation curves (i.e. without new exotic matter). It is then another argument to search a solution in the frame of the general relativity (i.e. without modifying the dynamics laws and without dark matter) and the purpose of the present work. Our study will focus on the equations of linearized general relativity, in the area of weak field (valid at the end of the galaxies) where the dark matter dominates.

\section{From General Relativity to Linearized General Relativity}

From general relativity, one deduces the linearized general relativity in the approximation of a quasi-flat Minkowski space $\left(g^{\mu v}=\eta^{\mu v}+h^{\mu v} ;\left|h^{\mu v}\right| \ll 1\right)$. With the following Lorentz gauge, it gives the following field equations as in [2]

(With $\square=\frac{1}{c^{2}} \frac{\partial^{2}}{\partial t^{2}}-\Delta$ ):

$$
\partial_{\mu} \bar{h}^{\mu v}=0 ; \square \bar{h}^{\mu v}=-2 \frac{8 \pi G}{c^{4}} T^{\mu v}
$$

With:

$$
\begin{gathered}
\bar{h}^{\mu v}=h^{\mu v}-\frac{1}{2} \eta^{\mu v} h ; h \equiv h_{\sigma}^{\sigma} ; h_{v}^{\mu}=\eta^{\mu \sigma} h_{\sigma v} ; \\
\bar{h}=-h
\end{gathered}
$$

The general solution of these equations is:

$$
\bar{h}^{\mu v}(c t, \boldsymbol{x})=-\frac{4 G}{c^{4}} \int \frac{T^{\mu v}(c t-|\boldsymbol{x}-\boldsymbol{y}|, \boldsymbol{y})}{|\boldsymbol{x}-\boldsymbol{y}|} \mathrm{d}^{3} \boldsymbol{y}
$$

In the approximation of a source with low speed, one has:

$$
T^{00}=\rho c^{2} ; T^{0 i}=c \rho u^{i} ; T^{i j}=\rho u^{i} u^{j}
$$

And for a stationary solution, one has:

$$
\bar{h}^{\mu v}(x)=-\frac{4 G}{c^{4}} \int \frac{T^{\mu v}(y)}{|x-y|} d^{3} y
$$

At this step, by proximity with electromagnetism, one traditionally defines a scalar potential $\varphi$ and a vector potential $H^{i}$. There are in the literature several definitions as in [3] for the vector potential $H^{i}$. In our study, we are going to define:

$$
\bar{h}^{00}=\frac{4 \varphi}{c^{2}} ; \quad \bar{h}^{0 i}=\frac{4 H^{i}}{c} ; \quad \bar{h}^{i j}=0
$$

With gravitational scalar potential $\varphi$ and gravitational vector potential $H^{i}$ :

$$
\begin{gathered}
\varphi(\boldsymbol{x}) \equiv-G \int \frac{\rho(\boldsymbol{y})}{|\boldsymbol{x}-\boldsymbol{y}|} \mathrm{d}^{3} \boldsymbol{y} \\
H^{i}(\boldsymbol{x}) \equiv-\frac{G}{c^{2}} \int \frac{\rho(\boldsymbol{y}) u^{i}(\boldsymbol{y})}{|\boldsymbol{x}-\boldsymbol{y}|} \mathrm{d}^{3} \boldsymbol{y}=-K^{-1} \int \frac{\rho(\boldsymbol{y}) u^{i}(\boldsymbol{y})}{|\boldsymbol{x}-\boldsymbol{y}|} \mathrm{d}^{3} \boldsymbol{y}
\end{gathered}
$$

With $K$ a new constant defined by: 


$$
G K=c^{2}
$$

This definition gives $K^{-1} \sim 7.4 \times 10^{-28}$ very small compare to $G$.

The field Equation (1) can be then written (Poisson equations):

$$
\Delta \varphi=4 \pi G \rho ; \Delta H^{i}=\frac{4 \pi G}{c^{2}} \rho u^{i}=4 \pi K^{-1} \rho u^{i}
$$

With the following definitions of $\boldsymbol{g}$ (gravity field) and $\boldsymbol{k}$ (gravitic field), those relations can be obtained from the following equations (also called gravitomagnetism):

$$
\begin{aligned}
& \boldsymbol{g}=-\boldsymbol{g r a d} \varphi ; \boldsymbol{k}=\boldsymbol{r o t} \boldsymbol{H} \\
& \operatorname{rot} \boldsymbol{g}=0 ; \operatorname{div} \boldsymbol{k}=0 ; \\
& \operatorname{div} \boldsymbol{g}=-4 \pi G \rho ; \operatorname{rot} \boldsymbol{k}=-4 \pi K^{-1} \boldsymbol{j}_{p}
\end{aligned}
$$

With the Equation (2), one has:

$$
h^{00}=h^{11}=h^{22}=h^{33}=\frac{2 \varphi}{c^{2}} ; h^{0 i}=\frac{4 H^{i}}{c} ; h^{i j}=0
$$

The equations of geodesics in the linear approximation give:

$$
\frac{\mathrm{d}^{2} x^{i}}{\mathrm{~d} t^{2}} \sim-\frac{1}{2} c^{2} \delta^{i j} \partial_{j} h_{00}-c \delta^{i k}\left(\partial_{k} h_{0 j}-\partial_{j} h_{0 k}\right) v^{j}
$$

It then leads to the movement equations:

$$
\frac{\mathrm{d}^{2} \boldsymbol{x}}{\mathrm{d} t^{2}} \sim-\operatorname{grad} \varphi+4 \boldsymbol{v} \wedge(\operatorname{rot} \boldsymbol{H})=\boldsymbol{g}+4 \boldsymbol{v} \wedge \boldsymbol{k}
$$

This is this relation that we are going to use to explain the observed results of [1]. All these relations can be retrieved starting with the parameterized post-Newtonian formalism with $k=\mathrm{B}_{\mathrm{g}} / 4$ (notation of [4]).

\section{From Linearized General Relativity to Rotation Curve at the End of the Galaxies}

The traditional computation of rotation speeds of galaxies consists in obtaining the force equilibrium from the three following components: the disk, the bugle and the halo of dark matter. More precisely, one has as in [5]:

$$
\frac{v^{2}(r)}{r}=\left(\frac{\partial \varphi(r)}{\partial r}\right) \text { with } \varphi=\varphi_{\text {disk }}+\varphi_{\text {bulge }}+\varphi_{\text {halo }}
$$

Or:

$$
\begin{aligned}
\frac{v^{2}(r)}{r} & =\left(\frac{\partial \varphi_{\text {disk }}(r)}{\partial r}\right)+\left(\frac{\partial \varphi_{\text {bulge }}(r)}{\partial r}\right)+\left(\frac{\partial \varphi_{\text {halo }}(r)}{\partial r}\right) \\
& =\frac{v_{\text {disk }}^{2}(r)}{r}+\frac{v_{\text {bulge }}^{2}(r)}{r}+\frac{v_{\text {halo }}^{2}(r)}{r}
\end{aligned}
$$

According to the linearized general relativity, the gravitational force is composed of the gravity fields (represented by $\partial \varphi_{\text {disk }}(r) / \partial r$ and $\partial \varphi_{\text {bulge }}(r) / \partial r$ in the previous equation) and by the gravitic field that we assume to be able to ex- 
plain the dark matter (represented by $\partial \varphi_{\text {halo }}(r) / \partial r$ ). Consequently, here, $\partial \varphi_{\text {halo }}(r) / \partial r$ gathers the gravitic force of all the components (disk, bulge, ...). This force due to the gravitic field $\boldsymbol{k}$ takes the following form $\left\|\boldsymbol{F}_{k}\right\|=m_{p} 4\|\boldsymbol{v} \wedge \boldsymbol{k}\|=m_{p} \partial \varphi_{\text {halo }}(r) / \partial r$ (from the Equation (13)). To simplify our computation, we idealize a situation where we have the approximation $\boldsymbol{v} \perp \boldsymbol{k}$. We can demonstrate that this perpendicularity is finally the more natural situation, meaning that this situation is very general, as demonstrated in [6].

This situation gives the following equation:

$$
\begin{aligned}
\frac{v^{2}(r)}{r} & =\left(\frac{\partial \varphi_{\text {disk }}(r)}{\partial r}\right)+\left(\frac{\partial \varphi_{\text {bulge }}(r)}{\partial r}\right)+4 k(r) v(r) \\
& =\frac{v_{\text {disk }}^{2}(r)}{r}+\frac{v_{\text {bulge }}^{2}(r)}{r}+4 k(r) v(r)
\end{aligned}
$$

Far from the center of the galaxies, when the gravitational field becomes negligible, the contribution of $\left(\partial \varphi_{\text {disk }}(r) / \partial r\right)+\left(\partial \varphi_{\text {bulge }}(r) / \partial r\right)$ is negligible. It is the area where dark matter dominates. So, far from the center of the galaxies, the equation becomes:

$$
\frac{v^{2}(r)}{r}=\frac{v_{\text {halo }}^{2}(r)}{r}=4 k(r) v(r)
$$

\section{From Theoretical to Observed Rotation Curve: A Uniform Gravitic Field $k_{0}$}

If we get back to the results of [1], they give the following equation:

$$
g_{\text {obs }}=\frac{v^{2}(r)}{r}=\frac{g_{\text {bar }}}{1-\mathrm{e}^{-\sqrt{g_{b a r} / g_{\dagger}}}}
$$

Let's see what happens where the dark matter dominates, i.e. at the end of the galaxies. This area is characterized by:

- very large value of $r \gg 15 \mathrm{kpc}$ (where the dark matter dominates);

- negligible gravity fields;

- low accelerations.

In this area, the results of [1] imply that we have:

$$
g_{\text {obs }} \propto \sqrt{g_{b a r}}
$$

By definition, $g_{b a r}=v_{b a r}^{2}(r) / r$, it then gives for the low accelerations:

$$
g_{o b s} \propto \frac{v_{b a r}(r)}{\sqrt{r}}
$$

Furthermore, for very large value of $r \gg 15 \mathrm{kpc}$, the curve $\sqrt{r}$ is extremely flat. It evolves very slowly and can be considered as constant with a very good approximation. It means that in this area the empirical relation can be written:

$$
g_{\text {obs }} \propto v_{b a r}(r)
$$

If we make the assumption that dark matter doesn't exist, at the ends of the galaxies the speed $v_{b a r}(r)$ is in fact the speed of the ordinary mass $v(r)$. One 
has then:

$$
g_{o b s} \propto v(r)
$$

The previous Equation (17) from the general relativity gives at the ends of the galaxies (where the effects of the bulge and disk fields are negligible):

$$
g_{\text {obs }}=\frac{v^{2}(r)}{r}=4 k(r) v(r)
$$

If we compare this relation with the empirical relation (22), the only way to conciliate them is to suppose that the gravitic field $k(r)$ at the ends of the galaxies is approximately constant. In other words, without dark matter and without modifying dynamic laws, to be in agreement with the observations, the general relativity implies the existence of an approximatively uniform gravitic field $k(r) \sim k_{0}$.

By this way, the general relativity verifies and explains the observations of [1], as we are going to see it, more in details.

\section{How This Uniform Gravitic Field $k_{0}$ Can Be Interpreted in General Relativity}

\subsection{A Uniform Gravitic Field $\boldsymbol{k}_{0}$ Coming from Clusters and Their Neighbors}

In fact, this solution has already been studied in [6]. Starting from others arguments and others observations, the article [6] deduced the same results, the existence of a residual uniform gravitic field $k_{0}$, and proposed an explanation for $k_{0}$. The uniform gravitic field $k_{0}$ would embed the galaxies and would come from the cluster of the galaxies and its neighbors. To understand how the uniform gravitic field works, one can think about a similar situation in the accelerators of particles, with the electromagnetism (I recall that the linearized general relativity is similar to the Maxwell's idealization as seen at the beginning). The particles follow circular trajectories with a uniform magnetic field and the source of the uniform magnetic field comes from structures of higher scale (the magnets). In the galaxies, the matter wouldn't escape because of this uniform gravitic field and the source would be the clusters. But as explained in [6], this uniform gravitic field cannot come from only one cluster (the field is too weak and cannot explain the extent of dark matter), it must be sustained by the neighboring clusters. One more time, one can understand it by thinking about a similar situation in the ferromagnetic matter with the electromagnetism. The spins are correlated to their neighbors, allowing sustaining a field from microscopic scale to a macroscopic scale, embedding the whole ferromagnetic matter.

\subsection{A Uniform Gravitic Field $\boldsymbol{k}_{0}$ with Predicted Values in Agreement with the Observations}

We are now going to see the accuracy of this solution. For the galaxy NGC 7331 (Figure 1), the theoretical study [6] gives for the gravitic field, the value $k_{0} \sim 10^{-16.3}$ at the ends of the galaxies with $v \sim 250 \mathrm{~km} \cdot \mathrm{s}^{-1}$. From the Equation (23), one can then deduce the expected observed value $g_{\text {obs }} \sim 10^{-10.3}$. This value 

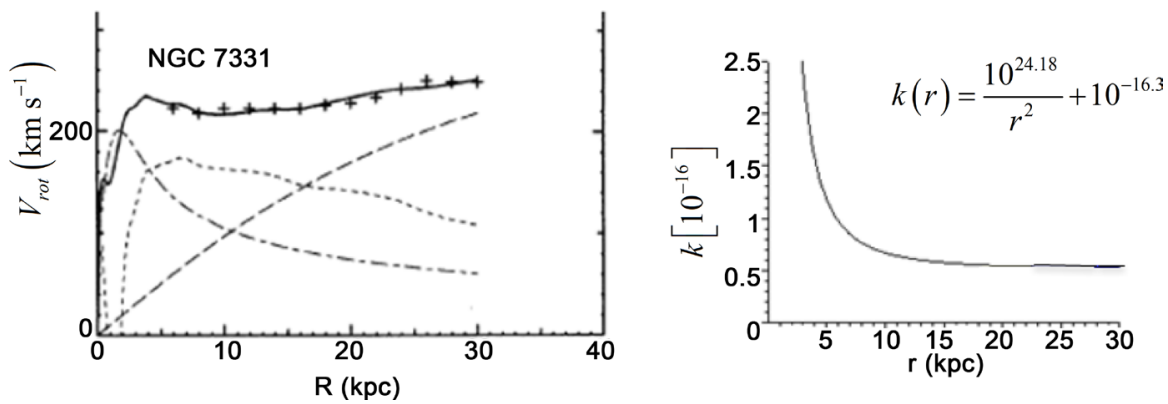

Figure 1. Example of rotation curves and gravitic fields for NGC 7331 galaxy. Left graphs are the measured data of the rotational speed from which the right graph is obtained $\left(k(r)=k_{1}(r)+k_{0}\right)$. Its analytic expression is given (Figure from [6]).

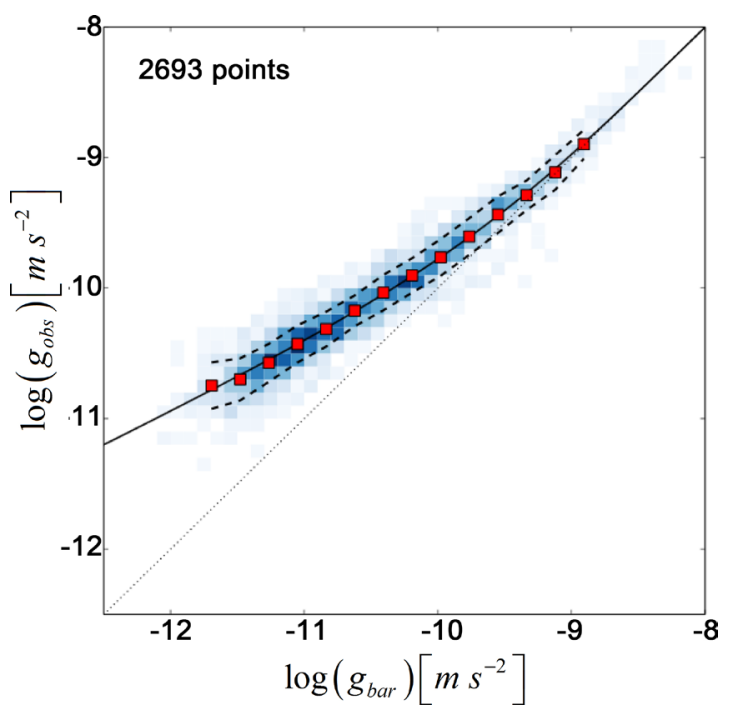

Figure 2. The centripetal acceleration observed in rotation curves, $g_{o b s}=v^{2} / r$, is plotted against that predicted for the observed distribution of baryons, $g_{\text {bar }}=\left|\partial \varphi_{\text {bar }} / \partial r\right|$. Nearly 2700 individual data points for 153 galaxies are shown in grayscale (Figure adapted with permission from [1]. Copyrighted by the American Physical Society).

is in agreement with the results of [1] (cf. Figure 2).

More precisely, all along the galaxy, we can write $k=k_{1}+k_{0}$ as in [6] with $k_{1}$ the own gravitic field of the galaxy and $k_{0}$ the uniform external gravitic field. As indicated in [6], inside the galaxy, for around $r<15 \mathrm{kpc}$, one has $k_{1} \gg k_{0}$ and then $k \sim k_{1}$ and for $r>15 \mathrm{kpc}$, one has $k_{1} \ll k_{0}$ and then $k \sim k_{0}$. It then implies that for $r<15 \mathrm{kpc}$ the own gravitic field of the galaxy acts, i.e. that $g_{b a r}$ depends on $k_{1}$ and for $r>15 \mathrm{kpc}$ the own gravitic field is progressively replaced by an external gravitic field, i.e. that $g_{b a r}$ depends little by little on $k_{0}$. It means that the solution of the general relativity implies a progressive change of nature of the correlation between $g_{o b s}$ and $g_{b a r}$ along the two areas. Concretely, for this latter area, $r>15 \mathrm{kpc}$, the article [6] gives $10^{-16.62}<k_{0}<10^{-16.3}$ and for the interval of rotation's speeds (at the ends of the galaxies where $k_{0}$ dominates) $50 \mathrm{~km} \cdot \mathrm{s}^{-1}<v<300 \mathrm{~km} \cdot \mathrm{s}^{-1}$.

In term of $g_{b a r}=4 k_{0} v$, it leads to the interval $10^{-11.32}<g_{b a r}<10^{-10.2}$. The 
solution in [6] expects then a different behavior in the correlation of $g_{\text {obs }}$ and $g_{b a r}$ in the interval $10^{-11.32}<g_{b a r}<10^{-10.2}$. The correlation's curve in [1] shows such a behavior (Figure 2). Indeed, near $g_{b a r} \sim 10^{-10}$, the correlation's curve starts deviating from the line of unity.

And one can focus on the remarkable agreement between the theoretical expectation and the experimental observations. The observed interval in [1] which deviates from the line of unity gives $10^{-11.7}<g_{b a r}<10^{-10}$ (obtained from 153 galaxies). The theoretical interval in [6] is obtained from only 16 galaxies. It represents near $70 \%$ of the observed interval with only $10 \%$ of the data.

One can also note that, as demonstrated in [6], the uniform gravitic field $k_{0}$ verifies the Poisson equation, just like the potential $g_{b a r}$ is a solution of the Poisson equation. Furthermore, the only way, in the frame of the general relativity, to modify the gravitational potential defined by the Poisson equation without modifying the distribution of the baryonic mass is to add a uniform gravitic field. The fact that the observations of [1] lead to satisfy this constraint, by conciliating the relations (22) and (23), is an important point that makes this solution very consistent.

\section{About Some Others Observed Predictions}

The fact that this gravitic field is uniform also means that it cannot come from the galaxy (otherwise it should decrease with the distance to the center). This result is in agreement with the simulations in [7]. Indeed these simulations demonstrate that the own gravitic field of the galaxy is negligible and cannot explain the dark matter. In this theoretical frame, only an external field can play a role to explain the dark matter. Furthermore, recent observations observed that the dark matter distribution is smoother than expected [8]. Our solution of an approximatively uniform $k_{0}$ which is imposed by general relativity is completely in agreement with these observations.

A recent publication [9] shows that ten billion years ago the galaxies were strongly dominated by baryonic matter. In other words, it seems not to have dark matter in and around the galaxies at this epoch. In the solution of [6], the gravitic field $k_{0}$ which explains the dark matter term should be generated by the clusters. But ten billion years ago, the clusters only began to appear. Consequently, the gravitic filed generated by these clusters and their neighbors should also only began to appear, meaning that without a well-structured cluster, the embedded gravitic field must not yet have is entire powerful. By this way, in the frame of this solution, dark matter should have little influence in the dynamic of the galaxies at this epoch. The observation of [9] would be consistent with this explanation of dark matter. It could then be a confirmation of the well-founded of this solution.

A more recent article [10] observes that the biggest, brightest galaxies in galaxy clusters have been heavily influenced by their unique environment since very early times. It is clearly what it is expected by the solution of the general re- 
lativity exposed in [6]. As previously indicated, $k_{0}$ should be generated from clusters and their neighbors. It means that just like the atomic spins of a ferromagnetic material tend to align under a magnetic field, the galaxies' "spin" should tend to align under a gravitic field. By this way, the neighboring galaxies should tend to align as observed. It is even one of the main predictions of the article [6].

Since the publication of [6], others observations have been published in agreement with its expected predictions:

1) The satellite dwarf galaxies are distributed according to plans;

2) For nearby galaxies, the plans of satellite dwarf galaxies must have the same orientation;

3) The plans must be aligned on the equatorial axis of the cluster they belong;

4) The clusters of neighboring galaxies must have a strong tendency to align;

5) A calculation of the order of magnitude provides that these alignments can extend over distances of tens of Mpc at least;

6) Statistically, the spin vectors of galaxies must be oriented in the same half-space (that of the cluster rotation vector).

The article [11] confirms the predictions 1, 2 and 3. The articles [12] and [10] confirm the predictions 4,5 and 6 . One also could mention the observation (totally unlikely if it was due to hazard) of 4 quasars perfectly aligned [13] or older papers [14] and [15] that revealed the alignment of quasars.

\section{Conclusions}

With the constraints of the observations of [1], the general relativity can explain the dark matter but only by a uniform gravitic field, external to the own gravitic field of the galaxy. This solution has been studied in [6]. It shows that this uniform gravitic field should come from the clusters of the galaxies and their neighbors. The agreement between the solution (allowed by the general relativity, without exotic matter and without modification of the dynamic's laws) and the observation [1] is total:

- The correlation between the baryonic mass distribution and the rotation's speed of the galaxies;

- the value of the gravitational potential;

- the fact that this potential is a solution of the Poisson equation;

- the relation of the potential in the weak accelerations $g_{\text {obs }} \propto \sqrt{g_{b a r}}$ (demonstrated with the general relativity);

- the characteristics of the correlation's curve (deviation from the line of unity for values smaller than around $g_{b a r} \sim 10^{-10}$ ).

Furthermore, we have seen that this solution also implied several unexpected predictions that have been recently verified:

- The satellite dwarf galaxies are distributed according to plans;

- For nearby galaxies, the plans of satellite dwarf galaxies must have the same orientation; 
- The plans must be aligned on the equatorial axis of the cluster they belong to;

- The clusters of neighboring galaxies must have a strong tendency to align;

- A calculation of the order of magnitude provides that these alignments can extend over distances of tens of Mpc at least;

- Statistically, the spin vectors of galaxies must be oriented in the same half-space (that of the cluster rotation vector);

- The galaxies of early clusters must be characterized by the absence of dark matter.

But this solution also implies others predictions not yet verified, in particular, a discrepancy in the measurement of the expected Earth's Lense-Thirring effect, as demonstrated in [16], of a value between around 0.3 and 0.6 milliarcsecond/ year, value inferior than the precision of "Gravity Probe B", the last experiments of measure of the Earth frame-dragging precession (39 mas/y) and of the geodetic effect (6606 mas/y). One can note that in this theoretical explanation, these experiments would be the more direct ways to measure the dark matter. This discrepancy is then a very important prediction of this solution that could be measured in the coming years (with experiments such as "GINGER").

One can also note that this solution could be adapted to explain the dark energy, another mystery of astrophysics more important than dark matter, as proposed in [17] and the result on the Hubble constant in [18] is in agreement with a prediction of this solution. But this adaptation implies an unexpected gravitational behavior between matter and antimatter, the gravitational interaction should be repulsive. The ongoing AEGIS and ALPHA experiments are testing this behavior.

All these first results, in agreement with the observations and general relativity, are to compare with the failure of the detection of the dark matter (as an exotic matter) until now. One can also focus on a remarkable consistency between this solution and the characteristic of dark matter. Indeed, the value of the gravitic field is so weak that it explains why dark matter has not been yet detected. And by the same time, because this term is so weak, its effect become measurable for large distance, as it can be seen with the Expression (23), explaining why dark matter appears only at large scale. Irony of history, dark matter and dark energy began to be seen as a potential failure of the general relativity, but with this solution, they would become two new great victories of general relativity. If it was the case, dark matter and dark energy will be certainly part of the top five of the most remarkable verifications of general relativity (just like space's curvature, gravitational waves and Lense-Thirring effect).

\section{References}

[1] McGaugh, S.S., Lelli, F. and Schombert, J.M. (2016) The Radial Acceleration Relation in Rotationally Supported Galaxies. Physical Review D, 117, 201101. https://arxiv.org/abs/1609.05917

[2] Hobson, M.P., Efstathiou, G.P. and Lasenby, A.N. (2006) General Relativity: An Introduction for Physicists. Cambridge University Press, Cambridge. 
https://doi.org/10.1017/CBO9780511790904

[3] Mashhoon, B. (2008) Gravitoelectromagnetism: A Brief Review. (Preprint) (arXiv:gr-qc/0311030). https://arxiv.org/abs/gr-qc/0311030

[4] Clifford, M.W. (2014) The Confrontation between General Relativity and Experiment. Living Reviews in Relativity, 17, 4.

https://link.springer.com/article/10.12942/lrr-2014-4 https://doi.org/10.12942//rr-2014-4

[5] Kent, S.M. (1986) Dark Matter in Spiral Galaxies. I-Galaxies with Optical Rotation Curves. Astronomical Journal, 91, 1301-1327. https://doi.org/10.1086/114106

[6] Le Corre, S. (2015a) Dark Matter, a New Proof of the Predictive Power of General Relativity. (Preprint) (arXiv:1503.07440). https://arxiv.org/abs/1503.07440

[7] Adamek, J., Daverio, D., Durrer, R. and Kunz, M. (2016) General Relativity and Cosmic Structure Formation. Nature Physics, 12, 346-349.

https://www.nature.com/nphys/journal/v12/n4/abs/nphys3673.html https://doi.org/10.1038/nphys3673

[8] Hildebrandt, et al. (2017) KiDS-450: Cosmological Parameter Constraints from Tomographic Weak Gravitational Lensing. Monthly Notices of the Royal Astronomical Society, 465, 1454-1498. https://doi.org/10.1093/mnras/stw2805

[9] Genzel, R., et al. (2017) Strongly Baryon-Dominated Disk Galaxies at the Peak of Galaxy Formation Ten Billion Years Ago. Nature, 543, 397-401.

https://www.nature.com/nature/journal/v543/n7645/full/nature21685.html https://doi.org/10.1038/nature21685

[10] West, M.J., De Propris, R., Bremer, M.N. and Phillipps, S. (2017) Ten Billion Years of Brightest Cluster Galaxy Alignments. Nature Astronomy, 1, Article Number: 0157. https://www.nature.com/articles/s41550-017-0157 https://doi.org/10.1038/s41550-017-0157

[11] Tully, B.R., et al. (2015) Two Planes of Satellites in the Centaurus a Group. The Astrophysical Journal Letters, 802, L25.

http://iopscience.iop.org/article/10.1088/2041-8205/802/2/L25

https://doi.org/10.1088/2041-8205/802/2/L25

[12] Taylor, A.R. and Jagannathan, P. (2016) Alignments of Radio Galaxies in Deep Radio Imaging of ELAIS N1. MNRAS, 459, L36-L40.

https://www.researchgate.net/publication/297897877_Alignments_of_radio_galaxie s_in_deep_radio_imaging_of_ELAIS_N1

https://doi.org/10.1093/mnrasl/slw038

[13] Hennawi, J.F., et al. (2015) Quasar Quartet Embedded in Giant Nebula Reveals Rare Massive Structure in Distant Universe. Science, 348, 779-783.

http://science.sciencemag.org/content/348/6236/779 https://doi.org/10.1126/science.aaa5397

[14] Hutsemekers, D. (1998) Evidence for Very Large-Scale Coherent Orientations of Quasar Polarization Vectors. Astronomy and Astrophysics, 332, 410-428. https://orbi.ulg.ac.be/bitstream/2268/3811/1/1998A\%2BA...332..410H.pdf

[15] Hutsemekers, D., et al. (2005) Mapping Extreme-Scale Alignments of Quasar Polarization Vectors. Astronomy and Astrophysics, 441, 915-930.

https://arxiv.org/abs/astro-ph/0507274 https://doi.org/10.1051/0004-6361:20053337

[16] Le Corre, S. (2016) Dark Matter: A Direct Detection. https://hal.archives-ouvertes.fr/hal-01276745/ 
[17] Le Corre, S. (2015) Dark Energy: A New Proof of the Predictive Power of General Relativity. https://hal-ens-lyon.archives-ouvertes.fr/ensl-01122689

[18] Riess, A.G., et al. (2016) A 2.4\% Determination of the Local Value of the Hubble Constant. The Astrophysical Journal, 826, 56.

https://iopscience.iop.org/article/10.3847/0004-637X/826/1/56

https://doi.org/10.3847/0004-637X/826/1/56

\section{Of Open Access Library}

Submit or recommend next manuscript to OALib Journal and we will provide best service for you:

- Publication frequency: Monthly

- 9 subject areas of science, technology and medicine

- Fair and rigorous peer-review system

- Fast publication process

- Article promotion in various social networking sites (LinkedIn, Facebook, Twitter, etc.)

- Maximum dissemination of your research work

Submit Your Paper Online: Click Here to Submit

Or Contact service@oalib.com 